\title{
USO DEL AFRECHO DE YUCA RACIONES DE PATOS CRIOLLOS EN CRECIMIENTO Y ENGORDE
}

Julio Rosales Conde * Augusta Tapullima**

\section{RESUMEN}

Una de las principales limitaciones para la crianza de patos es el alto costo y la poca disponibilidad de los insumos alimenticios. En la Amazonía contamos con muchos insumos cuyo uso en alimentación de patos es desconocido. El presente trabajo se realizó en el Centro Regional de Investigación del IIAP, filial Ucayali. El objetivo fue determinar los efectos biológicos y económicos de niveles de harina de hoja de yuca en alimentación de patos criollos mejorados en las fases de crecimiento y engorde. Se ensayaron cinco tratamientos con diferentes niveles de harina de hoja de yuca en la ración suministrada durante 42 días $(\mathrm{T} 1=\mathrm{O}, \mathrm{T} 2=1 \mathrm{O}$, $\mathrm{T} 3=20, \mathrm{~T} 4=30$ y $\mathrm{T} 5=40$ ). Se evaluó la ganancia de peso, consumo de alimento, conversión alimenticia y beneficio económico. El consumo de alimento diario (g/animal) fue similar $(\mathrm{P} \leq 05)$ entre tratamientos: $\mathrm{T} 1=147, \mathrm{~T} 2=149, \mathrm{~T} 3=136$, $\mathrm{T} 4=136$ y $\mathrm{T} 5=132$. Con relación a la ganancia de peso, se observó diferencia entre tratamientos $(\mathrm{P} \leq \mathrm{O}, 05)$, siendo los tratamientos $\mathrm{Ti}$ y $\mathrm{T} 2$ mayores que $\mathrm{T} 3$ y $\mathrm{T} 4$ y a su vez éstos mayores que el $T 5$, con estos incrementos diarios por animal: $\mathrm{Tl}=48$, $\mathrm{T} 2=50, \mathrm{~T} 3=47, \mathrm{~T} 4=46$ y $T 5=37 \mathrm{~g}$. Igualmente se observó diferencias $(\mathrm{P} \leq \mathrm{O}, 05)$ en conversión alimenticia, siendo $\mathrm{T} 5$ la de menor eficiencia alimenticia: $\mathrm{T} 1=3,12$, $\mathrm{T} 2=3,06, \mathrm{~T} 3=2,92, \mathrm{~T} 4=3, \mathrm{O} 1$ y $\mathrm{T} 5=3,59$. Económicamente el mejor beneficio neto fue obtenido en el T2. Se concluye que niveles de hasta $10 \%$ de harina de hoja de yuca en la ración no afectan los parámetros biológicos y económicos en la crianza de patos en crecimiento y engorde.

\footnotetext{
* Investigador del área Pecuaria del Centro Regional de Investigación del Instituto de Investigaciones de la Amazonía Peruana, filial Ucayali.

** Tesista de la Universidad Nacional Ucayali.
} 


\begin{abstract}
One of the limiting factors for the raising of ducks is the high cost and little availability of food products. The Amazonía counts with numerous food products whose use in the feeding of ducks is unknown. This work was done in the Centro Regional de Investigación of IIAP, in its Ucayali branch. Its objective was to determine the biological and economical effects of the levels of yucca flour in the feeding of native ducks improved in their growing and fattening phases. Five treatments were undertaken with different levels of yucca flour in the feed given the ducks throughout 42 days $(\mathrm{T} 1=0 ; \mathrm{T} 2=15 ; \mathrm{T} 3=30 ; \mathrm{T} 4=45$ and $\mathrm{T} 5=60)$. The weight gain the food consumption, the food conversion and the economical benefit were evaluated.
\end{abstract}

The daily food consumption (g/animal) was similar (P_05) up to levels of 30\% of yucca flour in the food, being $\mathrm{T} 1=165 ; \mathrm{T} 2=167 ; \mathrm{T} 3=171$; $\mathrm{T} 4=155$ and $\mathrm{T} 5=147$.

Regarding the weight gain no difference was observed among the treatments $(\mathrm{P} \leq 0,05)$ being the daily increases per animal: $\mathrm{T} 1=55 ; \mathrm{T} 2=57 ; \mathrm{T} 3=53 ; \mathrm{T} 4=50$ and $\mathrm{T} 5=48 \mathrm{~g}$.

Likewise, no difference was observed $(\mathrm{P} \leq 0,05)$ in the food conversion among treatments: $\mathrm{T} 1=3,11 ; \mathrm{T} 2=3,11 ; \mathrm{T} 3=3,35 ; \mathrm{T} 4=3,17$ and $\mathrm{T} 5=3,15$.

Economically, the best net benefit was obtained in T2.

We may conclude that levels up to $60 \%$ of yucca flour affected neither the weight increase nor the food efficiency; nevertheless, levels higher than $30 \%$ of yucca flour decreased the food consumption of ducks in the process of growth and fattening.

\title{
1. INTRODUCCION
}

La crianza de patos en la zona de Pucallpa se desarrolla principalmente a nivel familiar. Esta podría difundirse a nivel comercial, debido a sus buenas características:

rusticidad, fácil manejo, prolífico, buena ganancia de peso, adaptabilidad a diferentes condiciones climáticas y poco exigente en instalaciones.

Una de las limitaciones para la crianza comercial lo constituye el alto costo y la poca disponibilidad de insumos alimenticios tradicionales para la preparación de alimentos balanceados. El costo de alimentación en esta especie representa 
aproximadamente el $70 \%$ del total de los costos de producción. Una de las alternativas para disminuir costos es validar el uso de insumos regionales no tradicionales, que posean buenas características nutricionales y sean de bajo costo.

Durante el proceso de industrialización de la yuca, para la producción de harina se obtiene un subproducto llamado «afrecho», el cual es un insumo energético que podría reemplazar a otros insumos más costosos.

La incorporación del afrecho de yuca como insumo energético en la dieta de patos, en reemplazo parcial de otros insumos energéticos tradicionales, mejora los parámetros productivos y económicos de estos animales.

El objetivo de este estudio es determinar el efecto biológico y económico de niveles de afrecho de yuca en las raciones de patos en crecimiento y engorde.

\section{REVISLON DE LITERATURA}

Ríos (1973) menciona que en el procesamiento de las raíces de yuca para la obtención de harina se deriva un subproducto «afrecho», el cual es de bajo costo y fácil adquisición, pudiendo sustituir en la dieta alimenticia de los animales. Debido a su bajo costo y buena palatabilidad, el afrecho de yuca se puede utilizar como fuente energética para vacunos de leche, cerdos y aves, complementando los niveles de proteínas, grasas y minerales.

El contenido proteico del afrecho de yuca es bajo 2,720o (Rosales y Tang, 1995), siendo un factor nutricional limitativo, razón por la cual se requiere complementar con otros insumos proteicos tales como la harina de pescado, torta de soya, pasta de algodón, etc.; especialmente para mejorar los niveles de aminoácidos esenciales.

El afrecho y la harina de yuca, en términos de contenido de nutrientes, son similares.

Diferenciándose ligeramente el afrecho por su mayor contenido de fibra cruda $(6,12 \%)$ y por su menor proporción de extracto no nitrogenado $(87,430$ o) (Rosales y Tang, 1995).

El aporte energético del afrecho es menor que la harina de yuca. Para el caso de la harina de yuca sin cáscara 3,41 y para la harina de yuca con cáscara 3,43 Mcal/kg de EM (Agudu, 1979). 
San Martín (1993), probando el afrecho de yuca hasta niveles de 30\% en inicio y $40 \%$ en acabado de pollos de carne, no encontró diferencias $(\mathrm{P} \leq 0,05)$ en el incremento de peso con relación al control.

Bravo (1978), empleando el afrecho de yuca como sustituto del maíz en proporciones de $0,15,30,45$ y $60 \%$ en cerdos en crecimiento, no encontró diferencias $(\mathrm{P} \leq 0,05)$ en ganancia de peso y conversión alimenticia. La ganancia de peso diario lite: $610,650,670,650$ y $620 \mathrm{~g} /$ animal, y la conversión alimenticia de 2,54, 2,43, $2,17,2,47 \mathrm{y}$

2,37 , para los tratamientos con $0,15,30,45$ y $60 \%$ de afrecho de yuca en la ración, respectivamente.

Igualmente, Rosales y Urbietta (1993), usando el afrecho de yuca en niveles de 10, 35 y 6000 en la ración en cerdos cruzados Yorkshire x Landrace, en crecimiento y engorde, no encontraron diferencias $(\mathrm{P} \leq 0,05)$ en cuanto a ganancia de peso, consumo de alimento y conversión alimenticia. Al análisis económico, la ración con $60 \%$ de afrecho de yuca es la que reportó mayor beneficio económico.

Usando otro insumo similar, que es la harina de yuca en alimentación de polios de carne, Muller et al. (1974) y Vásquez et al. (1977) obtuvieron ganancias de peso similares a la testigo usando niveles de 10 y $15 \%$ de harina de yuca en la ración, respectivamente. Mediante la peletilización, es posible utilizar en mayores proporciones la harina de yuca. Así, Stevenson y Jackson obtuvieron rendimientos similares a la testigo, usando raciones con $50 \%$ de harina de yuca.

\section{MATERIALES Y METODOS}

Ubicación y duración del estudio. El estudio se realizó entre agosto y octubre de 1993, en la Estación Experimental «Villa Rica» del Centro Regional de Investigación del IIAP, filial Ucayali. Está ubicada en el km 12,4 de la carretera Federico Basadre (Pucallpa-Lima) y ubicada geográficamente a $8022^{\prime} 13 \mathrm{~S}$ y $74^{\circ} 34^{\prime} 23^{\prime \prime}$ '0 y a una altitud de $154 \mathrm{msnm}$.

Condiciones climáticas. La zona pertenece al ecosistema mayor de bosque tropical semi-siempreverde estacional (Cocbrane, 1982). La precipitación media anual es de $1852 \mathrm{~mm}$, temperatura media anual de 25,5 y una humedad relativa de $82 \%$. 
Instalaciones y equipos. Se usó dos pateras de $18 \mathrm{~m}^{2}$ cada una, construidas sobre un estanque piscícola, con techo de hoja de palma, piso y cerco enrejillado de madera, dividida en tres corrales de $6 \mathrm{~m}^{2}$ cada uno $(2 \times 3 \mathrm{~m})$, de los cuales se utilizaron cinco. Cada corral fue subdividido en dos subcorrales cada uno, equipados con comedero tipo tolva y un bebedero lineal automático.

Animales. Se usaron 50 patos criollos mejorados, 25 machos y 25 hembras de una edad de cuatro semanas y un peso promedio de $529 \mathrm{~kg}$. Cada grupo de patos de cada sexo fue distribuido al azar en cinco subgrupos, en los cuales fueron asignados los diversos tratamientos. Cada pato fue identificado mediante la colocación de bandas de aluminio en el ala.

Tratamientos. Cada subgrupo de cinco animales de cada sexo fue alimentado con una de cinco raciones isoproteicas e isoenergéticas, pero con diferentes niveles de afrecho de yuca, los que originaron cinco tratamientos:

$\mathrm{T} 1=$ Ración con $0 \%$ de afrecho de yuca.

$\mathrm{T} 2=$ Ración con 150 o de afrecho de yuca.

$\mathrm{T} 3=$ Ración con $30 \%$ de afrecho de yuca.

$\mathrm{T} 4=$ Ración con $45 \%$ de afrecho de yuca.

T5 $=$ Ración con $60 \%$ de afrecho de yuca.

Alimentación. Se alimentó desde la 4ta. hasta la 1Oma. semana de edad con raciones de 1600 de $\mathrm{PC}$ y $2,80 \mathrm{Mcal} / \mathrm{kg}$ de energía metabolizable (EM). Las fórmulas de las cinco dietas experimentales y las especificaciones nutricionales de las raciones se presentan en los Cuadros 1 y 2 . El análisis proximal de las raciones se muestra en el Cuadro 3. 
Cuadro 1. Fórmula de las cinco raciones experimentales para patos en crecimiento y engorde.

\begin{tabular}{|c|c|c|c|c|c|}
\hline \multicolumn{6}{|c|}{ Tratamientos } \\
\hline Insumo & $\mathrm{T} 1$ & $\mathrm{~T} 2$ & T3 & $\mathrm{T} 4$ & T5 \\
\hline \multicolumn{6}{|c|}{ 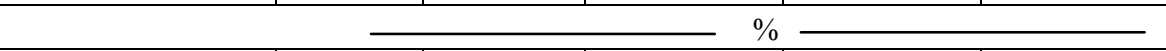 } \\
\hline Afrecho de yuca & 0,00 & 15,00 & 30,00 & 45,00 & 60,00 \\
\hline Maíz amarillo & 71,96 & 58,23 & 44,00 & 30,00 & 1,78 \\
\hline Polvillo de arroz & 11,41 & 8,48 & 5,57 & 2,64 & 8,54 \\
\hline Hna. de pescado I & 15,00 & 15,00 & 15,00 & 15,00 & 15,00 \\
\hline Torta de soya & 0,00 & 2,47 & 4,70 & 6,90 & 10,55 \\
\hline Carbonato de $\mathrm{Ca}$ & 1,04 & 0,23 & 0,15 & 0,06 & 0,00 \\
\hline Premezcla & 0,20 & 0,20 & 0,20 & 0,20 & 0,20 \\
\hline Cloruro de sodio & 0,39 & 0,39 & 0,38 & 0,18 & 0,00 \\
\hline Aceite vegetal & 0,00 & 0,00 & 0,00 & 0,02 & 3,93 \\
\hline Total & 100,00 & 100,00 & 100,00 & 100,00 & 100,00 \\
\hline
\end{tabular}

La alimentación fue ad libitum, la cual fue distribuida una vez al día a las 08:00 horas. El suministro de agua fue también ad libitum con agua de pozo.

\section{Cuadro2. Especificaciones nutricionales calculadas de las raciones para patos en crecimiento y engorde.}

\begin{tabular}{|l|r|r|r|r|r|}
\hline \multicolumn{7}{|c|}{ Tratamientos } \\
\hline Insumo & T1 & T2 & T3 & T4 & \multicolumn{1}{c|}{ T5 } \\
\hline \multicolumn{7}{|c|}{ EMaves } \\
\hline (Mcal/Kg) & 3,21 & 3,08 & 2,94 & 2,80 & 2,84 \\
Proteína (\%) & 16,15 & 15,37 & 16,21 & 15,49 & 15,52 \\
Fibra (\%) & 2,80 & 3,39 & 3,95 & 4,50 & 5,53 \\
Grasa (\%) & 6,35 & 5,46 & 4,54 & 3,65 & 6,41 \\
Lisina (\%) & 0,90 & 0,93 & 0,95 & 0,97 & 1,08 \\
Metionina (\%) & 0,40 & 0,39 & 0,37 & 0,36 & 0,37 \\
Met- Cist (\%) & 0,61 & 0,60 & 0,57 & 0,55 & 0,56 \\
Arginia (\%) & 0,92 & 0,93 & 0,93 & 0,94 & 1,02 \\
P disponible (\%) & 0,44 & 0,43 & 0,43 & 0,42 & 0,44 \\
Calcio (\%) & 0,97 & 0,70 & 0,70 & 0,70 & 0,76 \\
\hline
\end{tabular}


Cuadro 3. Análisis proximal ${ }^{1}$ en base seca de las raciones para patos en crecimiento y engorde.

\begin{tabular}{|l|r|r|r|r|r|}
\hline \multicolumn{7}{|c|}{ Tratamientos } \\
\hline Insumo & T1 & T2 & T3 & T4 & T5 \\
\cline { 1 - 5 } & \multicolumn{5}{|c|}{$\%$} \\
Proteína & 19,29 & 16,71 & 17,79 & 15,79 & 16,32 \\
Grasa & 6,30 & 6,81 & 2,39 & 5,99 & 6,36 \\
Fibra & 2,60 & 3,10 & 3,75 & 5,03 & 4,09 \\
Ceniza & 5,18 & 4,92 & 5,21 & 5,42 & 4,92 \\
Nifex & 66,63 & 68,46 & 70,86 & 67,77 & 68,31 \\
\hline
\end{tabular}

1 = Laboratorio de Socios y Tejidos Vegetales del INIA, Pucallpa.

\section{Variables Medidas}

a) Consumo de alimento. El control del consumo de alimento se realizó semanalmente, calculando la diferencia entre el alimento ofrecido y el alimento residual y desperdicio, obteniéndose de esta manera el consumo semanal y diario del alimento.

b) Ganancia de peso. El control de peso se realizó semanalmente en forma individual, desde el inicio hasta el final del experimento. Los pesos fueron tomados con los animales en ayunas en una balanza tipo reloj de $10 \mathrm{~kg}$ de capacidad con una aproximación de $50 \mathrm{~g}$.

e) Conversión alimenticia. La cantidad de alimento consumido por animal para ganar un kilo de peso vivo.

d) Análisis económico. Para el análisis económico se tuvo en cuenta los costos variables y costos fijos. Los costos variables de producción se obtuvieron de la suma de los costos de alimentación por animal en cada tratamiento. Para los costos fijos se gravó la compra de los patos, mano de obra, medicamentos, administración, depreciación de instalaciones y equipos e interés al capital. Los ingresos se estimaron multiplicando el precio de venta por kilo de peso vivo (PV) por el peso de cada animal. De la diferencia de los ingresos y el costo de producción se obtuvo la ganancia neta por animal. 
La ecuación usada para el beneficio neto fue la siguiente:

$\mathrm{E}=\mathrm{PYi}-(\mathrm{CVi} \pm \mathrm{CF})$

donde:

$\mathrm{B}=$ Beneficio neto en nuevos soles por patos

$\mathrm{i}=$ Tratamientos.

$\mathrm{P}=$ Precio por kilo de pato en nuevos soles.

$\mathrm{Yi}=$ Peso promedio final por pato en $\mathrm{kg}$.

$\mathrm{Cvi}=$ Costo variable por pato en nuevos soles.

$\mathrm{CF}=$ Costo fijo por pato en nuevos soles.

Diseño estadístico. Se usó el diseño estadístico Bloque Completamente Randomizado con dos repeticiones (Calzada, 1982), siendo el Modelo Aditivo Lineal:

$Y i j \mathrm{U}+\mathrm{ti}+\mathrm{Ej}+\mathrm{Eij}$

donde:

Yij =Valor observado para laj-ésima repetición del tratamiento i-ésimo.

$\mathrm{U}=$ Media poblacional.

ti $=$ Efecto del i-ésimo tratamiento.

Bj $=$ Efecto del j-ésimo bloque.

Eij $=$ Error experimental.

Para las comparaciones de los tratamientos se utilizó la prueba de significación de Duncan.

\section{RESULTADOS Y DISCUSION}

Consumo de alimento. El consumo de alimento de patos en las fases de crecimiento y engorde se presenta en el Cuadro 4. Allí se observa que el consumo de alimento disminuyó $(\mathrm{P} \leq 0,05)$ con niveles mayores de $30 \%$ de afrecho de yuca en la ración, debido posiblemente a la menor palatabilidad de la ración, por contener ésta mayor proporción de ácido cianhídrico, tal como afirma Buitrago (1990):

con los productos derivados de la yuca, cuyo contenido de ácidos cianhídrico es alto, los animales disminuyen drásticamente el consumo de alimento. 


\section{Cuadro 4. Consumo de alimento total y diario de patos en crecimiento y engorde1, en un periodo de 42 días. Promedio de dos repeticiones.}

\begin{tabular}{|l|c|c|}
\hline Tratamientos & $\begin{array}{c}\text { Consumo } \\
\text { Total }\end{array}$ & $\begin{array}{c}\text { Consumo } \\
\text { Diario }\end{array}$ \\
\hline T1 & $6,95 \mathrm{a}^{2}$ & 0,165 \\
T2 & $6,99 \mathrm{a}$ & 0,167 \\
T3 & $7,17 \mathrm{a}$ & 0,171 \\
T4 & $6,51 \mathrm{~b}$ & 0,155 \\
T5 & $6,16 \mathrm{c}$ & 0,147 \\
\hline
\end{tabular}

1/ Patos de cuatro semanas al inicio del experimento.

2/ Valores seguidos por letras iguales no difieren significativamente $(P \leq 0,05)$.

Ganancia de peso. Los pesos iniciales y finales, así corno la ganancia de peso durante la fase de crecimiento y la de engorde se muestran en el Cuadro 5. No se encontró diferencias $(\mathrm{P} \leq 0,05)$ en la ganancia de peso, debido a que las raciones con niVeles de afrecho de yuca fueron adecuadamente mezcladas con otros insumos proteicos, como la harina de pescado y la torta de soya (Cuadro 1), para conseguir raciones con similares tenores proteicos y energéticos que la ración testigo (Cuadros 2 y 3$)$.

\section{Cuadro 5. Peso inicial y ganancia diaria de peso de patos crecimientos y engorde1, en un periodo de 42 días. Promedio de dos repeticiones.}

\begin{tabular}{|l|r|r|r|r|}
\hline Tratamiento & \multicolumn{1}{|c|}{ Peso } & Ganancia de peso \\
\hline & \multicolumn{1}{|c|}{ Inicio } & \multicolumn{1}{|c|}{ Final } & \multicolumn{1}{c|}{ Total } & Diario \\
\hline & & & & 0,055 \\
T2 & 0,536 & 2,848 & $2,313 \mathrm{a}^{2}$ & 0,057 \\
T2 & 0,524 & 2,938 & $2,413 \mathrm{ab}$ & 0,053 \\
T3 & 0,514 & 2,727 & $2,213 \mathrm{~b}$ & 0,050 \\
T4 & 0,521 & 2,616 & $2,095 \mathrm{~b}$ & 0,048 \\
T5 & 0,551 & 2,515 & $1,995 \mathrm{c}$ & \\
\hline
\end{tabular}

1/ Patos de cuatro semanas de edad al inicio del experimento.

2/ Valores seguidos por letras iguales no difieren significativamente $(\mathrm{P} \leq \mathrm{O}, 05)$.

Resultado similar en alimentación de pollos de carne fue reportado por San Martín (1993), quien no encontró diferencias $(\mathrm{P} \leq 0,05)$ con relación a la testigo, hasta niveles de $3 \%$ en la fase de inicio y hasta $4 \%$ en la fase de acabado. 
Igualmente, pero en alimentación de cerdos en crecimiento, Bravo (1978) y Rosales y Urbietta (1993), reportaron que obtuvieron ganancias similares a la testigo usando raciones con $60 \%$ de afrecho de yuca.

Conversión alimenticia. La conversión alimenticia durante las fases de crecimiento y engorde, se muestra en el Cuadro 6. Se observa que la ración testigo y con $15 \%$ de afrecho de yuca fueron las de mejor eficiencia alimenticia, pero esta superioridad no fue significativa, debido a que el incremento de peso fue similar entre los tratamientos.

Cuadro 6. Conversión alimenticia de patos en crecimiento y engorde ${ }^{1}$, en un periodo de 42 días. Promedio de dos repeticiones.

\begin{tabular}{|l|c|c|c|}
\hline Tratamiento & $\begin{array}{c}\text { Consumo de } \\
\text { Alimento Total }\end{array}$ & $\begin{array}{c}\text { Ganancia de } \\
\text { Peso Total }\end{array}$ & $\begin{array}{c}\text { Conversión } \\
\text { Alimenticia }\end{array}$ \\
\hline T1 & 6,95 & 2,313 & $3,11 \mathrm{~b}^{2}$ \\
T2 & 6,99 & 2,413 & $3,11 \mathrm{a}$ \\
T3 & 7,17 & 2,213 & $3,35 \mathrm{a}$ \\
T4 & 6,51 & 2,095 & $3,17 \mathrm{a}$ \\
T5 & 6,16 & 1,995 & $3,15 \mathrm{a}$ \\
\hline
\end{tabular}

1/Patos de cuatro semanas de edad al inicio del experimento.

2 /Valores seguidos por letras iguales no difieren significativamente $(\mathrm{P} \leq \mathrm{O}, 05)$.

Análisis económico Para el. análisis económico se usaron los costos fijos y los costos variables, tal como se indica en los Cuadros 7 y 8 , respectivamente. El análisis de costo se realizó el mes de noviembre de 1993, teniendo como referencia un dólar igual a 2,18 nuevos soles.

Cuadro 7. Costos variables por pato por tratamiento (nuevos soles).

\begin{tabular}{|c|c|c|c|c|c|}
\hline VARIABLES & \multicolumn{3}{|c|}{ Tratamientos } & & \\
\hline & $\mathrm{T} 1$ & $\mathrm{~T} 2$ & T3 & $\mathrm{T} 4$ & $\mathrm{~T} 5$ \\
\hline $\begin{array}{l}\text { Consumo de alimento } \\
(\mathrm{Kg} / \text { animal) }\end{array}$ & 6,95 & 6,99 & 7,17 & 6,51 & 6,16 \\
\hline $\begin{array}{l}\text { Costo del alimento } \\
\text { (S/.Kg) }\end{array}$ & 0,518 & 0,502 & 0,484 & 0,467 & 0,497 \\
\hline $\begin{array}{l}\text { Costo Total del } \\
\text { Alimento (S/.) }\end{array}$ & 3,60 & 3,51 & 3,47 & 3,04 & 3,06 \\
\hline
\end{tabular}


Cuadro 8. Costo fijo por pato por tratamiento (nuevos soles).

\begin{tabular}{ll}
\hline Variables & S/. \\
\hline Pato & 3,50 \\
Sanidad & 0,12 \\
Mano de obra & 0,27 \\
Depreciación de inst. y equipos & 0,05 \\
\hline Total & 3,92 \\
\hline
\end{tabular}

El análisis económico que se realizó al final del estudio se muestra en el Cuadro 9, observándose que el mejor beneficio se obtuvo usando $15 \%$ de afrecho de yuca en la ración, donde en relación a la testigo fue mayor en 7,60\%. Este mayor beneficio se debió principalmente al mayor incremento de peso y menor costo del alimento en el T2, por contener dentro de su composición un insumo más económico, como lo es el afrecho de yuca.

Cuadro 9. Beneficio promedio neto por animal por tratamiento.

\begin{tabular}{|l|r|r|r|r|r|r|r|}
\hline Tra. & \multicolumn{1}{c|}{ P } & Y (Kg) & PxY & CV & CF & \multicolumn{2}{c|}{$\begin{array}{c}\text { Beneficio Neto } \\
\text { Animal Kg Carne }\end{array}$} \\
\hline 1 & 4,0 & 2,848 & 11,39 & 3,60 & 3,92 & 3,87 & 1,359 \\
\hline 2 & 4,0 & 2,938 & 11,75 & 3,51 & 3,92 & 4,32 & 1,470 \\
\hline 3 & 4,0 & 2,727 & 10,91 & 3,47 & 3,92 & 3,52 & 1,291 \\
\hline 4 & 4,0 & 2,616 & 10,46 & 3,04 & 3,92 & 3,50 & 1,338 \\
\hline 5 & 4,0 & 2,515 & 10,06 & 3,06 & 3,92 & 3,08 & 1,225 \\
\hline
\end{tabular}

$\mathrm{P} \quad=$ Precio por kilo de pato en nuevos soles.

$\mathrm{Y} \quad=$ Peso promedio de pato por tratamiento en $\mathrm{kg}$.

$\mathrm{CV}=$ Costo variable de pato por tratamiento en nuevos soles.

$\mathrm{CF}=$ Costo fijo de pato por tratamiento en nuevos soles. 


\section{CONCLUSIONES}

De los resultados obtenidos en el presente estudio, se derivan las siguientes conclusiones:

1. La utilización del afrecho de yuca hasta niveles de $60 \%$ reemplazando parcialmente al maíz y al polvillo de arroz, no afectó la ganancia de peso ni la eficiencia alimenticia. Sin embargo, con niveles mayores de 30\% de afrecho de yuca, el consumo de alimento se vio disminuido en los patos en crecimiento y engorde.

2. El mayor beneficio económico se obtuvo con el reemplazo parcial, $15 \%$ de afrecho de yuca (T2), siendo ésta mayor en $7,6 \%$ con respecto a la testigo.

\section{BIBLIOGRAFIA}

AGUDU, E. W. 1979. An evaluation of cassava (Manihot esculenta Crantz) as a dietary ingredientsfor chicks. Tesis Ph.D. College Park University of Maryland. $81 \mathrm{pp}$.

BRAVO, M. 1978. Utilización de diferentes niveles de afrecho de yuca en raciones para cerdos en crecimiento en la zona de Tingo María. Tesis Ing. Zootecnista. Universidad Nacional Agraria de la Selva. Tingo María, Perú. 41 pp.

BUITRAGO, J. 1990. La yuca en la alimentación animal. Cali (Colombia): Centro Internacional de Agricultura Tropical (CIAT). 446 pp.

CALZADA, J. 1982. Métodos Estadísticos para la Investigación Científica. Lima: 3ra. edición. 546 pp.

COCHRANE, T. T. 1982. Caracterización agroecológica para el desarrollo de pasturas en suelos ácidos en América Tropical. En: Toledo, J. M. (ed) Manual para la Evaluación Agronómica, Cali (Colombia): Red Internacional de Pastos Tropicales, CIAT. 23-44 pp.

MULLER, Z.; CHOU, K. y NAH, K. 1974. Cassava as a total substitute for cereals in livestock and poultry rations. En: World Animal Review 12(1):1924. 
RIOS, B. 1973. Digestibilidad del afrecho de yuca en ovinos. Tesis Ing. Zootecnista. Universidad Nacional Agraria de la Selva. Tingo María. 41 pp.

ROSALES, J. M.; TANG T. 1995. Análisis proximal y digestibilidad in vitro de los insumos alimenticios de la zona de Ucayali. Por publicar en: Folia Amazónica, Instituto de Investigaciones de la Amazonía Peruana. Iquitos (Perú).

ROSALES, J. M.; URBIETTA, H. 1993. Comparativo de niveles de afrecho de yuca en raciones para cerdos en crecimiento y engorde, en la zona de Pucallpa. En:

Folia Amazónica, y. 5, Nro. 1 y 2, Instituto de Investigaciones de la Amazonía Peruana. Iquitos (Perú): 207 pp.

SAN MARTIN, M. 1993. Uso del subproducto de la harina de yuca para pollos de carne. Tesis Ing. Zootecnista. Universidad Nacional Agraria, La Molina, Lima.

STEVENSON, M. 1984. The nutritional value of dried cassava root meal in broiler diets. En: J Sci. Food Agric. 34: 1361-1367.

VASQUEZ, F.; ARTEAGA, C.; AVILA, E. 1977. Harina de yuca (Manihot esculenta) en dietas para pollos de engorde y gallinas en postura. En: Tea. Pec. México. 32:53-57. 